\title{
Análise de correlação entre habilidade de Resolução de Problemas e desempenho em disciplinas de programação
}

\author{
Jhonatas Costa Oliveira, Leandro S. G. Carvalho, \\ Elaine H. T. Oliveira, David B. F. Oliveira \\ Universidade Federal do Amazonas \\ Manaus - AM, Brasil \\ \{jco,galvao,elaine,david\}@icomp.ufam.edu.br
}

Apesar de estudado há décadas e, mesmo com avanços, a literatura ainda não chegou a uma conclusão sobre o que caracteriza um bom resolvedor de problemas [13]. Como levantado por Veerasamy et al. [14], muitos alunos ingressantes no ensino superior possuem habilidades limitadas de resolução de problemas e enfrentam dificuldade em utilizar os principais conceitos de programação para expressar suas soluções em código. Com efeito, é importante analisar a relação entre habilidade de resolução de problemas e o desempenho em disciplinas de programação, a fim de que sejam adotadas intervenções precoces proativamente $[1-5,8-11]$.

Na Universidade Federal do Amazonas (UFAM), a disciplina de Introdução à Programação de Computadores (IPC) é ofertada como componente curricular obrigatório de 17 cursos de graduação nonmajor. Porém, a taxa de aprovação nessa disciplina, entre 2010 e 2019 , oscilou entre $40 \%$ a $50 \%$. Isso representa um desperdício de recursos públicos na formação de profissionais de nível superior. Como uma forma de lidar com esse problema, este trabalho em progresso tem por objetivo validar e aplicar um questionário diagnóstico que potencialmente identifique alunos com dificuldade na resolução de problemas e com consequente alto risco de reprovação. Mais especificamente, o questionário será usado como instrumento preditor, através da análise de correlação entre os fatores avaliados e o desempenho do aluno, possibilitando acompanhá-lo de forma especial para que os riscos de reprovação sejam reduzidos na disciplina de IPC. Note que desenvolver questionários de avaliação desse tipo é um grande desafio na área de educação em computação [12]. Com isso, uma alternativa é adaptar instrumentos existentes [5].

Nesse sentido, Lishinski e colegas [5] observaram que a capacidade de resolução de problemas se correlaciona significativamente com o desempenho nas tarefas de programação (elaboração de código), mas não se correlaciona com o desempenho nos exames de múltipla escolha. Na UFAM, tipicamente, os exercícios e avaliações em IPC envolvem justamente a habilidade de elaboração de código. Portanto, é importante verificar se os resultados observados no exterior se aplicam no contexto local.

Além disso, o Programa Internacional de Avaliação de Estudantes (PISA - Programme for International Student Assessment) é um estudo comparativo internacional, realizado a cada três anos pela Organização para a Cooperação e Desenvolvimento Econômico

Fica permitido ao(s) autor(es) ou a terceiros a reprodução ou distribuição, em parte ou no todo, do material extraído dessa obra, de forma verbatim, adaptada ou remixada, bem como a criação ou produção a partir do conteúdo dessa obra, para fins não comerciais, desde que sejam atribuídos os devidos créditos à criação original, sob os termos da licença CC BY-NC 4.0.

EduComp'21, Abril 26-30, 2021, Jataí, Goiás, Brasil (On-line)

(C) 2021 Copyright mantido pelo(s) autor(es). Direitos de publicação licenciados à Sociedade Brasileira de Computação (SBC).

\author{
Filipe Dwan Pereira \\ Universidade Federal de Roraima \\ Boa Vista - RR, Brasil \\ filipe.dwan@ufrr.br
}

(OCDE). A avaliação do PISA não envolve apenas a memorização de conhecimentos, mas a habilidade do estudante em matemática, leitura, ciências e resolução de problemas [6]. Em 2003, a principal área avaliada foi Resolução de Problemas. Do teste de 2003, foram liberados ao público dez questões de Resolução de Problemas, sendo que 4 dessas questões foram utilizadas por [5]. Dos itens utilizados, dois são de análise e projeto de sistema, um de tomada de decisão e outro de solução de problemas. A escolha foi baseada no nível de dificuldade e relevância do problema, que foram identificadas calculando a porcentagem dos alunos que responderam corretamente aos itens no teste do PISA 2003.

Com isso, neste estudo, o instrumento usado por [5] será adaptado para o português brasileiro, a fim de aplicá-lo aos alunos de IPC da UFAM e verificar a correlação com o desempenho nessa disciplina, confirmando ou não os achados de Lishinski e colegas. Obtendo-se valores lineares nos fatores avaliados no instrumento, será utilizado o coeficiente de correlação de Pearson, o mesmo utilizado por Lishinski e colegas. Se as variáveis tenderem a mover-se na mesma direção relativa, mas não necessariamente a uma taxa constante, será aplicado o coeficiente de correlação de Spearman, conforme recomendado em [14]. Note que os autores [5] correlacionaram a pontuação de habilidades de resolução de problemas (PSS, em inglês) do aluno com o seu desempenho em Programação Introdutória e, concluem que o PSS é um preditor significativo para estimar o desempenho do aluno.

Neste trabalho em andamento, a minuta de questionário será traduzida da Língua Inglesa para a Língua Portuguesa e passará pelo primeiro processo de validação. O segundo processo da validação será marcado por entrevistas, com alunos de graduação da UFAM, no modelo Think Aloud, seguindo o método usado por [7], que validará a coesão e coerência da minuta. Após as entrevistas, a minuta passará por correções de inconsistências destacadas nas entrevistas. Posteriormente, o questionário será aplicado aos alunos de uma turma teste, que irão responder o questionário e darão sugestões de mudança, caso haja. Adiante serão encontrados padrões nas respostas discursivas a fim de transformá-las em questões objetivas. Por fim, passará pelo último processo de entrevistas para a validação do questionário final.

Após o encerramento da validação, o questionário será aplicado em diversas turmas de IPC, no início do período letivo, por meio da plataforma de juiz online CodeBench ${ }^{1}$. As respostas dadas pelos alunos aos itens do questionário serão coletadas e, em seguida, correlacionadas com a nota obtida em IPC durante o mesmo período letivo. Os resultados obtidos pelo presente estudo serão contrastados com aqueles obtidos por Lishinski e colegas [5].

\footnotetext{
${ }^{1}$ codebench.icomp.ufam.edu.br/
} 


\section{AGRADECIMENTOS}

Os autores agradecem ao apoio e financiamento prestado pela Universidade Federal do Amazonas - UFAM por meio do Edital 081/2019 - PROPESP/UFAM, do Programa Institucional de Bolsas de Iniciação Científica (PIBIC). Além disso, esta pesquisa, realizada no âmbito do Projeto Samsung-UFAM de Ensino e Pesquisa (SUPER), de acordo com o artigo 48 do Decreto no 6.008/2006 (SUFRAMA), foi parcialmente financiada pela Samsung Electronics da Amazônia Ltda., nos termos de Lei Federal no 8.387/1991, mediante contrato 001/2020, firmado com a Universidade Federal do Amazonas e a FAEPI, Brasil. Contamos também com o apoio da Coordenação de Aperfeiçoamento de Pessoal de Nível Superior - Brasil (CAPES) Código de Financiamento 001 e do Conselho Nacional de Desenvolvimento Científico e Tecnológico - Brasil (Processo 308513/2020-7).

\section{REFERÊNCIAS}

[1] Ada Araujo, Daniel Lopes Zordan Filho, Elaine Harada Teixeira de Oliveira, Leandro Silva Galvão de Carvalho, Filipe Dwan Pereira, and David Braga Fernandes de Oliveira. 2021. Mapeamento e análise empírica de misconceptions comuns em avaliações de introdução à programação. In Anais do Simpósio Brasileiro de Educação em Computação. SBC, 123-131.

[2] Ingrid Lima dos Santos, David Braga Fernandes Oliveira, Leandro Silva Galvão de Carvalho, Filipe Dwan Pereira, and Elaine Harada Teixeira de Oliveira. 2020. Tempos de Transição em Estados de Corretude e Erro como Indicadores de Desempenho em Juízes Online. In Anais do XXXI Simpósio Brasileiro de Informática na Educação. SBC, 1283-1292.

[3] Filipe Dwan, Elaine Oliveira, and David Fernandes. 2017. Predição de zona de aprendizagem de alunos de introdução à programação em ambientes de correção automática de código. In Brazilian Symposium on Computers in Education (Simpósio Brasileiro de Informática na Educação-SBIE), Vol. 28. 1507.

[4] Samuel Fonseca, Elaine Oliveira, Filipe Pereira, David Fernandes, and Leandro Silva Galvão de Carvalho. 2019. Adaptação de um método preditivo para inferir o desempenho de alunos de programação. In Brazilian Symposium on Computers in Education (Simpósio Brasileiro de Informática na Educação-SBIE), Vol. 30. 1651

[5] Alex Lishinski, Aman Yadav, Richard Enbody, and Jon Good. 2016. The influence of problem solving abilities on students' performance on different assessment tasks in CS1. In Proceedings of the 47th ACM technical symposium on computing science education. 329-334.

[6] Organização para a Cooperação e Desenvolvimento Econômico (OCDE). 2005. PISA 2003 Technical Report. Disponível em http://www.oecd.org/education/ school/programmeforinternationalstudentassessmentpisa/35188570.pdf (fev/2021).

[7] Miranda C Parker, Mark Guzdial, and Shelly Engleman. 2016. Replication, validation, and use of a language independent CS1 knowledge assessment. In Proceedings of the 2016 ACM conference on international computing education research. 93-101.

[8] Filipe Pereira, Elaine Oliveira, David Fernandes, Hermino Junior, and Leandro Silva Galvão de Carvalho. 2019. Otimização e automação da predição precoce do desempenho de alunos que utilizam juízes online: uma abordagem com algoritmo genético. In Brazilian Symposium on Computers in Education (Simpósio Brasileiro de Informática na Educação-SBIE), Vol. 30. 1451.

[9] Filipe Dwan Pereira, Samuel C Fonseca, Elaine HT Oliveira, David BF Oliveira, Alexandra I Cristea, and Leandro SG Carvalho. 2020. Deep learning for early performance prediction of introductory programming students: a comparative and explanatory study. Brazilian journal of computers in education. 28 (2020), 723-749.

[10] F. D. Pereira, E. H. Oliveira, D. Fernandes, and A. Cristea. 2019. Early performance prediction for CS1 course students using a combination of machine learning and an evolutionary algorithm. In 2019 IEEE 19th International Conference on Advanced Learning Technologies (ICALT), Vol. 2161. IEEE, 183-184.

[11] Filipe D Pereira, Elaine HT Oliveira, David BF Oliveira, Alexandra I Cristea, Leandro SG Carvalho, Samuel C Fonseca, Armando Toda, and Seiji Isotani. 2020. Using learning analytics in the Amazonas: understanding students' behaviour in introductory programming. British fournal of Educational Technology (2020).

[12] A. V. Robins. 2019. Novice programmers and introductory programming. In The Cambridge Handbook of Computing Education Research. Cambridge University Press, Cambridge, Chapter 12, 327-376.

[13] Isabel Vale, Teresa Pimentel, and Ana Barbosa. 2015. Ensinar matemática com resolução de problemas. Quadrante 24, 2 (2015), 39-60.

[14] Ashok Kumar Veerasamy, Daryl D’Souza, Rolf Lindén, and Mikko-Jussi Laakso. 2019. Relationship between perceived problem-solving skills and academic performance of novice learners in introductory programming courses. Fournal of Computer Assisted Learning 35, 2 (2019), 246-255. 